\title{
ARTICLE
}

Myelodysplastic syndrome

\section{Recurrent somatic mutations are rare in patients with cryptic dyskeratosis congenita}

\author{
Martin Kirschner ${ }^{1} \cdot$ Angela Maurer $^{1} \cdot$ Marcin W. Wlodarski $\mathbb{D}^{2} \cdot$ Monica S. Ventura Ferreira ${ }^{1} \cdot$ Anne-Sophie Bouillon ${ }^{1} \cdot$ \\ Insa Halfmeyer ${ }^{1} \cdot$ Wolfgang Blau $^{3} \cdot$ Michael Kreuter $^{4}$ - Martin Rosewich ${ }^{5}$. Selim Corbacioglu ${ }^{6}$. Joachim Beck ${ }^{7}$. \\ Michaela Schwarz ${ }^{8}$. Jörg Bittenbring ${ }^{9} \cdot$ Markus P. Radsak $^{7} \cdot$ Christian Matthias Wilk $^{10} \cdot$ Steffen Koschmieder $^{1}$. \\ Matthias Begemann ${ }^{11} \cdot$ Ingo Kurth $^{11} \cdot$ Mirle Schemionek $^{1} \cdot$ Tim H. Bümmendorf $^{1} \cdot$ Fabian Beier $^{1}$
}

Received: 18 December 2017 / Revised: 11 March 2018 / Accepted: 15 March 2018 / Published online: 2 April 2018

(c) Macmillan Publishers Limited, part of Springer Nature 2018

\begin{abstract}
Dyskeratosis congenita (DKC) is a paradigmatic telomere disorder characterized by substantial and premature telomere shortening, bone marrow failure, and a dramatically increased risk of developing myelodysplastic syndrome (MDS) or acute myeloid leukemia (AML). DKC can occur as a late-onset, so-called cryptic form, with first manifestation in adults. Somatic MDS-related mutations are found in up to $35 \%$ of patients with acquired aplastic anemia (AA), especially in patients with short telomeres. The aim of our study was to investigate whether cryptic DKC is associated with an increased incidence of MDS-related somatic mutations, thereby linking the accelerated telomere shortening with the increased risk of MDS/AML. Samples from 15 adult patients (median age: 42 years, range: 23-60 years) with molecularly confirmed cryptic DKC were screened using next-generation gene panel sequencing to detect MDS-related somatic variants. Only one of the 15 patients (7\%) demonstrated a clinically relevant MDS-related somatic variant. This incidence was dramatically lower than formerly described in acquired AA. Based on our data, we conclude that clonal evolution of subclones carrying MDS-related mutations is not the predominant mechanism for MDS/AML initiation in adult cryptic DKC patients.
\end{abstract}

These authors contributed equally: Tim H. Brümmendorf, Fabian Beier.

Electronic supplementary material The online version of this article (https://doi.org/10.1038/s41375-018-0125-x) contains supplementary material, which is available to authorized users.

Fabian Beier

fbeier@ukaachen.de

1 Department of Hematology, Oncology, Hemostaseology and Stem Cell Transplantation, Medical Faculty, RWTH Aachen University, Aachen, Germany

2 Department of Pediatric Hematology, Oncology and Stem Cell Transplantation, University of Hospital Freiburg, Freiburg, Germany

3 Department of Hematology and Oncology, Justus-Liebig University, Giessen, Germany

4 Center for Interstitial and Rare Lung Diseases, Pneumology and Respiratory Critical Care Medicine, Thoraxklinik, University of Heidelberg, Heidelberg, Germany

5 Department of Paediatric Pulmonology, Allergy and Cystic Fibrosis, Children's Hospital, Goethe-University, Frankfurt, Germany

\section{Introduction}

The telomere length (TL) shortens with each cell division and reflects the replicative history of a cell or tissue [1,2]. Telomere shortening (TS) can be slowed down or reversed by the enzyme telomerase-whereas, impaired telomerase function leads to accelerated TS [3]. In analogy to double-

6 Department of Pediatric Hematology, Oncology and Stem Cell Transplantation, University Hospital Regensburg,

Regensburg, Germany

7 Department of Hematology, Medical Oncology, \& Pneumology, University Medical Center of the Johannes Gutenberg University, Mainz, Germany

8 Department of Hematology and Oncology, University Hospital Charité, Berlin, Germany

9 Department of Hematology and Oncology, University Hospital Saarland, Homburg, Germany

10 Hematology, University Hospital Zurich and University of Zurich, Zurich, Switzerland

11 Institute of Human Genetics, Medical Faculty, RWTH Aachen University, Aachen, Germany 
strand breaks, the critically short telomeres trigger DNA damage checkpoints and as a consequence, the affected cells undergo replicative senescence as long as these checkpoints are intact. Further rounds of replication and/or functional impairment of the respective checkpoints result in increasing chromosomal instability and eventually, clonal evolution $[1,2]$. Thus, critical shortening of telomeres can both act as a predisposing factor for malignant transformation or be a part of a tumor suppressor mechanism $[4,5]$.

Dyskeratosis congenita (DKC) is a paradigmatic disorder to study the effects of critically short telomeres. Classical DKC is characterized clinically by mucocutaneous features, development of bone marrow failure (BMF) and critically short telomeres [6]. In addition, DKC patients are faced with an increased incidence of acute myeloid leukemia (AML), myelodysplastic syndrome (MDS), and solid tumors, e.g., head-neck squamous cell carcinoma [7]. In DKC patients, the risk of developing secondary AML or MDS is increased 200-fold and 2.500-fold, respectively, compared to the normal population with both malignancies typically occurring at a median age of $\sim 35$ years [8]. Premature TS is frequently caused by mutations in genes affecting telomerase or telomerase-related components, leading to functionally reduced telomerase activity. In $30-40 \%$ of all clinical DKC cases with short telomeres, an underlying genetic defect cannot be identified thereby complicating the correct diagnosis, especially in BMF patients without further clinical symptoms [9]. While classical DKC is typically diagnosed in childhood, a second late-onset, so-called cryptic subtype of DKC with diverging non-hematologic stigmata often first manifests itself in early or middle-aged adulthood [10].

Detection of short telomeres with recurrent somatic mutations is a hallmark of various acquired clonal disorders, such as MDS [2, 11, 12], CML [13], or others [2]. In addition, in otherwise healthy individuals, clonal hematopoiesis of indeterminate potential (CHIP) with detection of gene mutations originally described in myeloid neoplasms was found to be associated with an increased risk for hematological cancers $[14,15]$. Moreover, somatic mutations are observed in up to $35 \%$ of patients with acquired aplastic anemia (AA), most likely due to a growth advantage of clonal cells with acquired mutations in the context of an immune escape from the autoimmune-mediated attack against the hematopoietic stem cell (HSC) compartment [16]. This concept was also corroborated by the fact that mutations were preferentially found in patients with premature TS $[17,18]$.

In this study, we aimed to investigate whether accelerated TS, due to impaired telomere maintenance in adult patients with molecularly confirmed cryptic DKC, was associated with an increased frequency of MDS-related somatic mutations reflecting the first step of the multi-step leukemogenesis, explaining the remarkably increased risk of MDS and/or AML observed in this late-onset hereditary BMF syndrome.

\section{Methods and patients}

Analysis included 15 patients from the "Aachen Telomeropathy Registry" $(n=13)$ and the Freiburg pediatric bone marrow failure registry $(n=2)$. Informed consent was obtained of all patients. All patients had clinical features typical of DKC. No patient had cytologic signs of MDS. The median age of the cohort was 42 years (range: $23-60$ y). All patients had confirmed mutations in DKC-causing genes (TERC $n=7$, TERT $n=7, D K C 1 n=1$, Table 1 ). Additional clinical details of two patients with MDS/AML are shown in Supplementary Table 1. For TL measurement, flow-FISH was used according to previously described protocols, and TL is indicated in kilobases $(\mathrm{kb})$ [19-22]. A self-customized next-generation sequencing (NGS) panel ("telomere-panel") for known DKC-causing genes was used to identify genetic variants [23]. The NGS was carried out on the gDNA of the peripheral blood samples of all 15 patients due to the hypoplastic/aplastic bone marrow, except for patient \#4, where sufficient bone marrow for additional analysis was available. In addition, we used a clinically validated self-customized NGS-panel ("MDS/MPN-panel") including genes harboring mutations, which are typically associated with myeloid neoplasms (ABL, ASXL1, BARD, CALR, CBL, CEBPA, CHEK2, CSF3R, DNMT3A, ETNK1, ETV6, EZH2, IDH1, IDH2, JAK2, KIT, KRAS, MPL, NFE2, NRAS, PDGFRA, PTPN11, RUNX1, SETBP1, SF3A1, SF3B1, SH3B2 (LNK), SRSF2, TCF12, TET2, TP53, and $U 2 A F 1)$. For detailed information see Supplementary Material and Methods.

\section{Results}

TL was found to be below the first percentile in peripheral blood lymphocytes in 12 out 15 patients (Fig. 1a). the mean TL was $4.78 \mathrm{~kb} \pm 0.63 \mathrm{SD}$ in the lymphocyte and $5.42 \mathrm{~kb} \pm 1.01 \mathrm{SD}$ in the granulocyte (Fig. 1b) subpopulation, respectively. Using the "Telomere-panel", all detected mutations showed a 50/50 ratio between the reference and the mutated allele in line with the anticipated heterozygosity of the detected mutations (Table 1). The "MDS/MPNpanel" sequencing revealed a relevant somatic MDS-related mutation in only 1 out of 15 patients analyzed. This patient (\#7) harbored a mutation in the U2AF1 gene $(\mathrm{c} .101 \mathrm{C}>\mathrm{T}$, p.Ser34Phe; allele frequency $16 \%$ ), which has been described in MDS [24], in addition to the known DKCcausing TERT mutation (c.2915 G>A; p.Arg972His) 


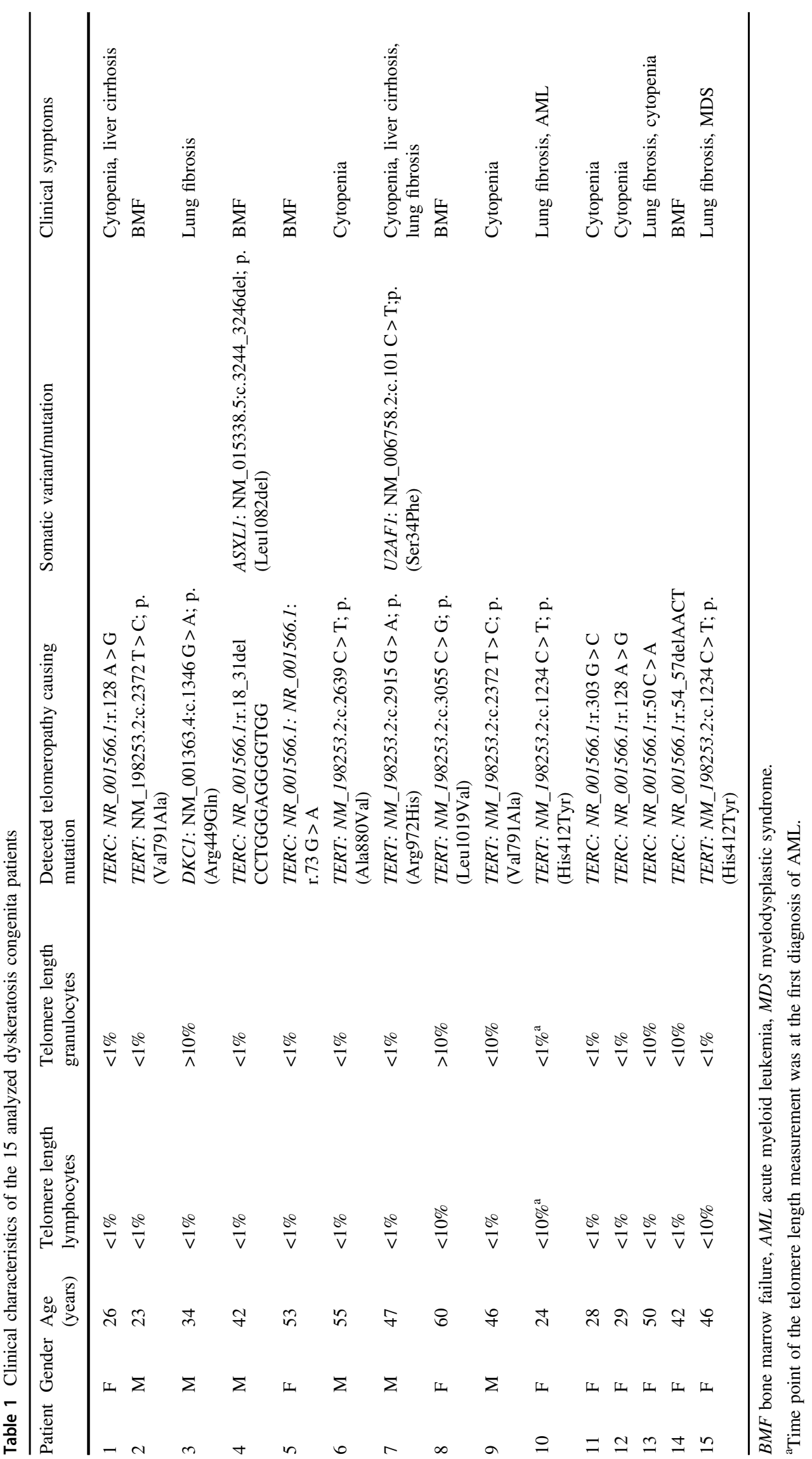


Fig. 1 Telomere analysis and detailed presentation of the observed MDS-related mutations: Lymphocyte (a) and granulocyte (b) telomere length of 15 dyskeratosis congenita patients, $1 \%, 10 \%, 50 \%, 90 \%$, and $99 \%$ percentile are indicated. Patient \#4 with the detected ASXL1 mutation is depicted in red. Patient \#7 with the detected U2AF1 mutation is depicted in green. c Detailed depiction of the detected inframe ASXL1 deletion and U2AF1 mutation. MDS myelodysplastic syndrome, $\mathrm{kb}$ kilobases

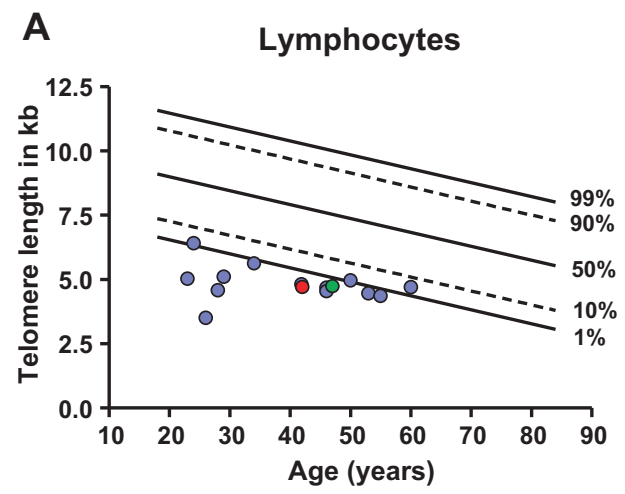

B

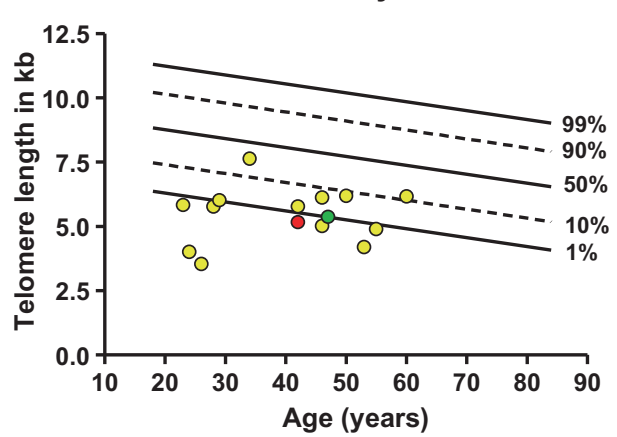

C

ASXL1

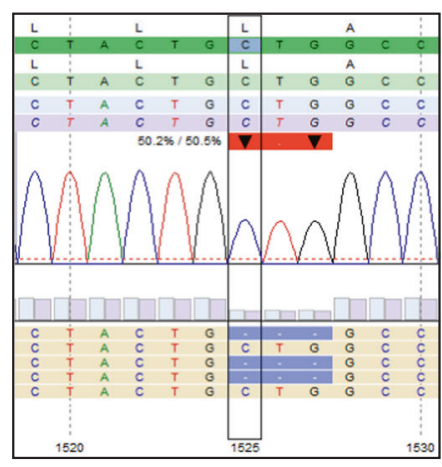

U2AF1

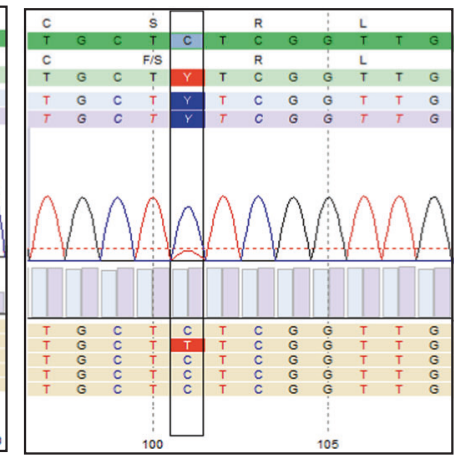

(Fig. 1c). In another patient with an inherited TERC mutation (patient \#4), an ASXL1 variant (c.3244_3246CTGdel; freq.: 48\% [4708/9347]; p.Leu1082del) (Fig. 1c) was detected in the peripheral blood. A similar allele frequency was also observed by analyzing the respective bone-marrow sample (freq.: 50\%). Interestingly, TS in granulocytes was not further accelerated in the patients carrying the described variant (patient \#4: 5,17 kb) and mutation (patient \#7: 5,38 $\mathrm{kb})$, as compared to the remaining 13 patients of the cohort $(5.54 \mathrm{~kb}, p=0.84, t$-test $)$.

\section{Discussion}

This is the first report of a systematic analysis of somatic mutations in the rare cohort of adult patients with late-onset, cryptic DKC. Until now, no data existed on the exact mechanism for tumor development in DKC patients. The occurrence and potential accumulation of MDS-related clonal mutations might represent a possible mechanism underlying secondary MDS and/or subsequent AML development [25]. However, to our surprise, we observed characteristic acquired mutations in typical MDS-linked genes in only 1 out of 15 patients present in our cohort. The detected ASXL1 variant is most likely a rare germline variant (allele frequency in the sample about 50\%) of uncertain significance (see clinvar-including bioinformatical analysis, mean allele frequency /dbsnp150 rs754183801: $0.002 \%)$.

Based on our data, clonal hematopoiesis with detection of MDS-related genes is a rather rare event in adult DKC patients, compared to other myeloid diseases with short telomeres, but functional (compared to DKC) telomerase complex (e.g., typical elderly MDS or acquired AA) [11, 12, 16-18]. Therefore surprisingly, evolution of subclones of HSC carrying MDS-related clonal mutations does not seem to be the predominant mechanism for MDS/AML initiation in cryptic DKC patients.

At this stage, the pathophysiology underlying the malignant transformation in (cryptic) DKC remains unclear. Various murine models favor the role of chromosomal instability due to critically short telomeres as the main contributing factor for tumor initiation [1]. In line with this observation, both analyzed patients with MDS or AML (pat. \#10 and \#15) show chromosomal aberrations but no MDS-related mutations.

We hypothesize that significant replicative potential is a prerequisite of HSCs that can undergo multi-step clonal evolution from genetically normal HSCs via CHIP and eventually toward malignant transformation into MDS and/ or AML. In comparison, in patients with DKC, due to the already accelerated TS, the replicative reserve in the HSC compartment is typically limited. Therefore, upon a rather limited number of cell divisions, the DKC stem cells either 


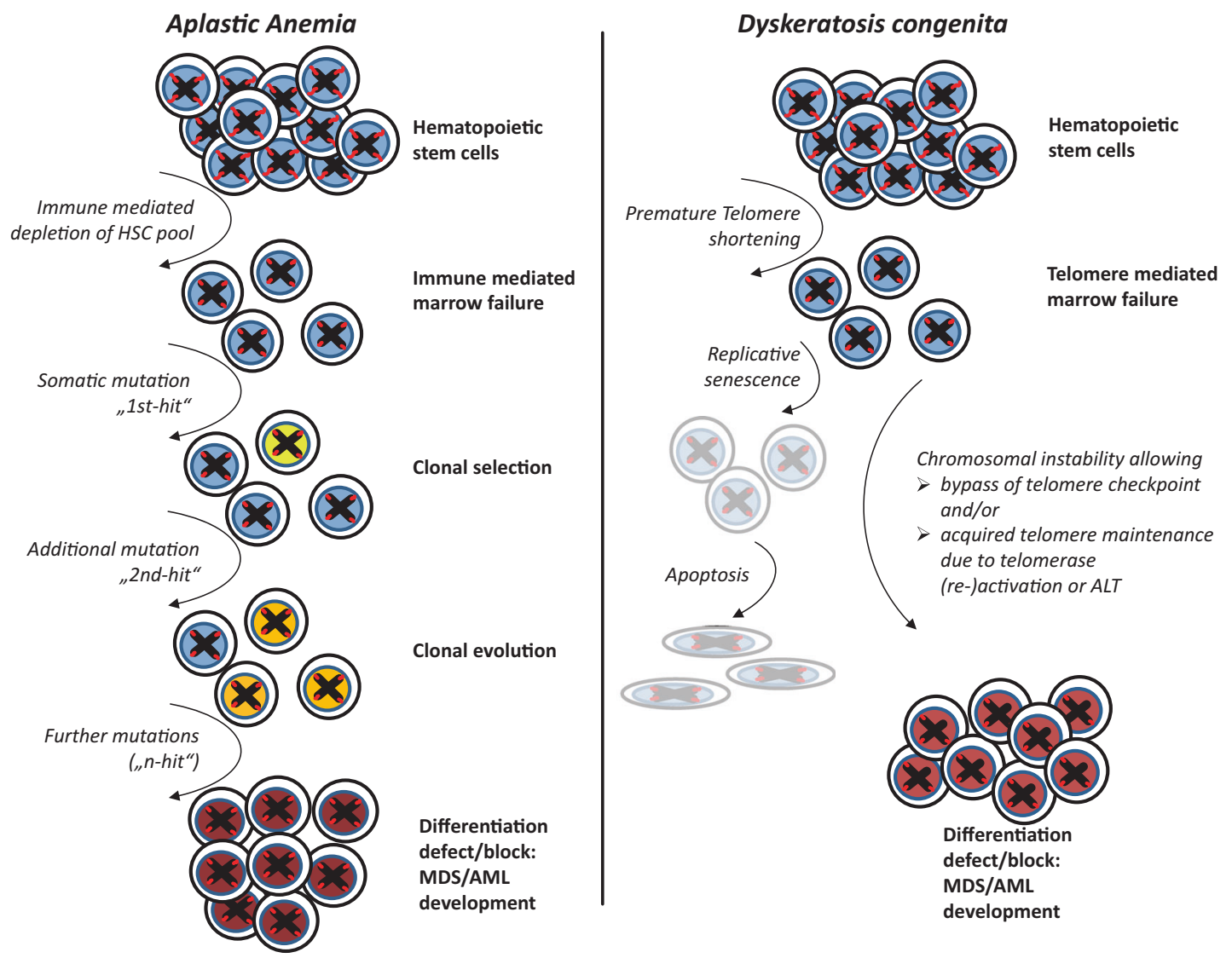

Fig. 2 Schematic representation of possible MDS/AML development in aplastic anemia and dyskeratosis congenita. ALT alternative lengthening of telomeres, AML acute myeloid leukemia, MDS myelodysplastic syndrome, HSC hematopoietic stem cells

undergo replicative senescence/apoptosis, or alternatively acquire the defined genetic events that allow them to either bypass the telomere checkpoint, or maintain or (re-)elongate TL early enough to become clonally selected. The latter is achieved either by reactivating the telomerase activity or alternative lengthening of telomeres (ALT, Fig. 2).

U2AF1 mutations lead to increased proliferation of hematopoietic progenitors [26], thus providing a possible escape mechanism to allow HSC to circumvent the telomere-mediated replicative senescence. Genetic mosaicism, somatic gene reversion [27, 28], occurrence of hTERT promotor mutations, or epigenetic factors (e.g., genomic imprinting) may be the possible alternative mechanisms to allow clonal expansion in the presence of short telomeres in this patient. However, detailed follow-up studies are needed to further elucidate the mechanism of how cells carrying the impaired telomerase activity actually manage to expand to a significant clone size.

Finally, we hypothesize that the absence of typical somatic mutations in NGS screening might potentially help to discriminate the clinically overt DKC patients without DKC-causing mutations from patients with acquired AA typically carrying MDS-related mutations at high frequency. Overall, since correct diagnosis of DKC is of utmost importance for treatment (including donor selection in the case of stem cell transplantation) and management of complications [9], significant clinical benefits can be expected from better identification of particularly late-onset hereditary BMF syndromes, such as cryptic DKC.

We here provide the first data showing that additional somatic mutations in DKC are rare events, possibly arguing against the multi-step clonal evolution based on sequentially acquired MDS-related mutations as the predominant mechanism for the development of secondary hematologic malignancies in adult and late-onset DKC. Due to the uncertain incidence and probable underdiagnosis of this disease subgroup in adults, prospective and cross-registry validation of these results and systematic follow-up of affected patients need to be initiated to further substantiate these findings.

Acknowledgements We are thankful to Lucia Vankann, Kristina Feldberg and Stephan Klinkenberg for their excellent technical assistance. We thank Kim Kricheldorf for the excellent administration of the "Aachen Telomeropathy Registry". This work was partly supported by a grant from "Lichterzellen e.V." (THB). 
Author contributions MK: Performed the experiments, analyzed and interpreted the data and wrote the manuscript. AM: Performed the experiments and analyzed the data. MW: Provided patient samples, clinical data and interpreted the data. MSVF, A-SB, IH: Performed the experiments and interpreted the data. WB, MK, MR, SC, JB, MS, JB, MPR, CMW: Provided patient samples, clinical data and interpreted the data. SK, MB, IK, MS: Performed the experiments, analyzed and interpreted the data. THB co-planned the study, analyzed and interpreted the data and wrote the manuscript; FB conceived and planned the study design, interpreted the data, and wrote the manuscript.

\section{Compliance with ethical standards}

Conflict of interest The authors declare that they have no conflict of interest.

\section{References}

1. Blasco MA. Telomeres and human disease: ageing, cancer and beyond. Nat Rev Genet. 2005;6:611-22.

2. Brummendorf $\mathrm{TH}$, Balabanov S. Telomere length dynamics in normal hematopoiesis and in disease states characterized by increased stem cell turnover. Leukemia. 2006;20:1706-16.

3. Greider CW, Blackburn EH. Identification of a specific telomere terminal transferase activity in tetrahymena extracts. Cell. 1985;43:405-13.

4. Cooper JN, Young NS. Clonality in context: hematopoietic clones in their marrow environment. Blood. 2017;130:2363-72.

5. Martinez P, Blasco MA. Telomere-driven diseases and telomeretargeting therapies. J Cell Biol. 2017;216:875-87.

6. Fernandez Garcia MS, Teruya-Feldstein J. The diagnosis and treatment of dyskeratosis congenita: a review. J Blood Med. 2014;5:157-67.

7. Alter BP, Giri N, Savage SA, Rosenberg PS. Cancer in the National Cancer Institute inherited bone marrow failure syndrome cohort after 15 years of follow-up. Haematologica. 2017;103:30-39.

8. Alter BP, Giri N, Savage SA, Rosenberg PS. Cancer in dyskeratosis congenita. Blood. 2009;113:6549-57.

9. Townsley DM, Dumitriu B, Young NS. Bone marrow failure and the telomeropathies. Blood. 2014;124:2775-83.

10. Schmitt K, Beier F, Panse J, Brummendorf TH. (Pan-)cytopenia as first manifestation of kryptic telomeropathies in adults. Dtsch Med Wochenschr. 2016;141:1578-80.

11. Bejar R, Stevenson K, Abdel-Wahab O, Galili N, Nilsson B, Garcia-Manero G, et al. Clinical effect of point mutations in myelodysplastic syndromes. N Engl J Med. 2011;364:2496-506.

12. Sperling AS, Gibson CJ, Ebert BL. The genetics of myelodysplastic syndrome: from clonal haematopoiesis to secondary leukaemia. Nat Rev Cancer. 2017;17:5-19.

13. Brummendorf TH, Holyoake TL, Rufer N, Barnett MJ, Schulzer M, Eaves CJ, et al. Prognostic implications of differences in telomere length between normal and malignant cells from patients with chronic myeloid leukemia measured by flow cytometry. Blood. 2000;95:1883-90.
14. Jaiswal S, Fontanillas P, Flannick J, Manning A, Grauman PV, Mar BG, et al. Age-related clonal hematopoiesis associated with adverse outcomes. N Engl J Med. 2014;371:2488-98.

15. Steensma DP, Bejar R, Jaiswal S, Lindsley RC, Sekeres MA, Hasserjian RP, et al. Clonal hematopoiesis of indeterminate potential and its distinction from myelodysplastic syndromes. Blood. 2015;126:9-16.

16. Stanley N, Olson TS, Babushok DV. Recent advances in understanding clonal haematopoiesis in aplastic anaemia. Br J Haematol. 2017;177:509-25.

17. Park HS, Park SN, Im K, Kim SM, Kim JA, Hwang SM, et al. Telomere length and somatic mutations in correlation with response to immunosuppressive treatment in aplastic anaemia. $\mathrm{Br}$ J Haematol. 2017;178:603-15.

18. Kulasekararaj AG, Jiang J, Smith AE, Mohamedali AM, Mian S, Gandhi S, et al. Somatic mutations identify a subgroup of aplastic anemia patients who progress to myelodysplastic syndrome. Blood. 2014;124:2698-704.

19. Beier F, Balabanov S, Buckley T, Dietz K, Hartmann U, Rojewski $\mathrm{M}$, et al. Accelerated telomere shortening in glycosylphosphatidylinositol (GPI)-negative compared with GPI-positive granulocytes from patients with paroxysmal nocturnal hemoglobinuria (PNH) detected by proaerolysin flow-FISH. Blood. 2005;106:531-3.

20. Werner B, Beier F, Hummel S, Balabanov S, Lassay L, Orlikowsky $\mathrm{T}$, et al. Reconstructing the in vivo dynamics of hematopoietic stem cells from telomere length distributions. eLife 2015;4:e08687.

21. Beier F, Masouleh BK, Buesche G, Ventura Ferreira MS, Schneider RK, Ziegler P, et al. Telomere dynamics in patients with del (5q) MDS before and under treatment with lenalidomide. Leuk Res. 2015 S0145-2126(15)30380-5.

22. Brummendorf TH, Maciejewski JP, Mak J, Young NS, Lansdorp PM. Telomere length in leukocyte subpopulations of patients with aplastic anemia. Blood. 2001;97:895-900.

23. Weidner CI, Lin Q, Birkhofer C, Gerstenmaier U, Kaifie A, Kirschner M, et al. DNA methylation in PRDM8 is indicative for dyskeratosis congenita. Oncotarget. 2016;7:10765-72.

24. Graubert TA, Shen D, Ding L, Okeyo-Owuor T, Lunn CL, Shao J, et al. Recurrent mutations in the U2AF1 splicing factor in myelodysplastic syndromes. Nat Genet. 2011;44:53-57.

25. Walter MJ, Shen D, Ding L, Shao J, Koboldt DC, Chen K, et al. Clonal architecture of secondary acute myeloid leukemia. N Engl J Med. 2012;366:1090-8.

26. Shirai CL, Ley JN, White BS, Kim S, Tibbitts J, Shao J, et al. Mutant U2AF1 expression alters hematopoiesis and pre-mRNA splicing in vivo. Cancer Cell. 2015;27:631-43.

27. Jongmans MC, Verwiel ET, Heijdra Y, Vulliamy T, Kamping EJ, Hehir-Kwa JY, et al. Revertant somatic mosaicism by mitotic recombination in dyskeratosis congenita. Am J Hum Genet. 2012;90:426-33.

28. Perdigones N, Perin JC, Schiano I, Nicholas P, Biegel JA, Mason $\mathrm{PJ}$, et al. Clonal hematopoiesis in patients with dyskeratosis congenita. Am J Hematol. 2016;91:1227-33. 